\title{
Call your doctor: prospective description study of telemedicine during the first COVID-19 outbreak in a Swiss primary care practice
}

Joëlle Suillot

Department of Internal Medicine, Fribourg Hospital, Switzerland

Sophie Zuercher

La Tour medical practice, 1635 La Tour-de-Trême, Switzerland

Lydie Zufferey

University of Fribourg, Switzerland

Julien Sagez ( $\nabla$ js@cm-latour.ch )

La Tour medical practice, 1635 La Tour-de-Trême, Switzerland https://orcid.org/0000-0002-9807-9540

\section{Research Article}

Keywords: Telemedicine, COVID-19, primary care, private practice

Posted Date: November 16th, 2020

DOI: https://doi.org/10.21203/rs.3.rs-108798/v1

License: (9) This work is licensed under a Creative Commons Attribution 4.0 International License.

Read Full License 


\section{Abstract}

Background: Telemedicine is a healthcare assistance method which has been promoted during the COVID-19 pandemic for the management of patients. The aim of this study is to quantify the type of request that primary care physicians are experiencing, as well as the healthcare assistance modalities and ultimately, how many physical consultations are necessary.

Methods: We conducted a prospective, descriptive study in a primary care practice in Switzerland on telephone consultations related to COVID-19. The data collected included the reasons for the call, symptoms, healthcare assistance modalities and follow-up. The categorical variables are expressed as percentages of the total cases or groups.

Results: We included 200 calls corresponding to 113 patients. The majority of patients $(76.1 \%)$ were taken care of solely through telemedicine. The physical consultations were due to the need: for a swab test $(12.4 \%)$, for a somatic assessment at the practice $(9.7 \%)$ and for an emergency services consultation (1.8\%). In $64.6 \%$ of cases, patient assistance required only one phone call. Calls lasted 7.85 minutes on average and were more frequent on Mondays (24\%) and Fridays (18.5\%). Calls led to the prescription of medication in $12.5 \%$ of cases, of a bronchodilator in $3 \%$ of cases and of an antibiotic in $0.5 \%$ of cases.

Conclusion: A rigorous telephone follow-up strategy carried out by primary care physicians requires few physical consultations in patients showing symptoms of COVID-19.

\section{Background}

The emergence of the COVID-19 virus in December 2019 in Wuhan in China, then its spread to reach the pandemic stage, declared in March 2020, caused millions of respiratory infections (1). Due to the rapid development of the disease, unprecedented public health measures were put in place and changes in hospital structures were required (2).

Outpatient medicine and primary care practices were also immediately required to implement new assistance strategies, in particular telemedicine $(3,4)$. Primary care medicine has been faced with a double challenge: having to deal with a considerable number of patient requests and, at the same time, having to restrict the movement of contagious patients $(5,6)$.

In such circumstances, we carried out this descriptive study, with the objective of quantifying the type of requests primary care physicians have to deal with, the modalities of assistance, the prescription of medication in telemedicine and finally, how many physical consultations are necessary. As a secondary objective, we gathered the patient symptoms.

\section{Methods}


This is an observational, prospective, monocentric study at a multidisciplinary private practice medical centre in Bulle. Bulle is a rural area in French-speaking Switzerland and was hit by COVID-19 in early April. The study took place from 6 April to 28 May 2020. The medical centre includes six general practitioners, two assistant physicians in general medicine, a paediatrician, a specialist in pain relief, two rheumatologists and several paramedic staff. The number of patients followed in the medical centre, in terms of adult general medicine, is approximately 6,700. Telemedicine consultations were not carried out at the medical centre before April 2020.

The criteria for inclusion and exclusion in the study are shown in Table 1.

For each call, the age and gender of the patient were recorded, as well as the characteristics of the call, symptom description and assistance offered. The data collected during the telephone interviews is shown in Table 2. The choice of symptoms was based on the published literature (7). Five general internal medicine general practitioners, two assistant physicians in general internal medicine as well as a pain relief specialist anaesthesiologist took part in the study. Data collection was carried out using a semi-structured interview sheet. In order to ensure the consistency of data, a glossary including the definitions of each item and the recommendations in force was put in place and regularly updated. The choice of the modality of assistance was left to each physician, according to their assessment of the circumstances. Patients showing symptoms were called back between D7-D10 to check on their health.

Therefore, the assistance offered to patients was adapted based on the recommendations of local public health authorities. Informed consent was obtained with each call. The data was collected anonymously. The study obtained authorisation from the Ethics Committee (Project 2020-00595). All methods were performed in accordance with the relevant guidelines and regulations.

The statistical analysis was performed on SPSS, version 26, (Cary, NC) with the support of descriptive tests. The continuous variables are expressed as means and standard deviations (SD). The categorical variables are expressed as percentages of the total cases or groups.

\section{Results}

\section{Description of the telephone calls}

During the study period, 200 telephone calls were considered, corresponding to 113 patients. The average age was 44 years old and the median age 42 years old. The patients were $68.1 \%$ women and $31.9 \%$ men. Figure 1 shows the number of calls per patient and the reason for the call. The telephone calls were $55 \%$ follow-ups, $22 \%$ initial assessments of ill patients and $23 \%$ for advice. A telephone follow-up consultation was organised for $48 \%$ of the patients, a consultation only where necessary was agreed for $52 \%$ of the patients. Patient assistance required 1 telephone call in $64.6 \%$ of cases, 2 telephone calls in $17.7 \%$ of cases, 3 telephone calls in $9.7 \%$ of cases and more than 3 telephone calls in $8 \%$ of cases. 
Of the 113 patients taken care of, $76.1 \%$ were assisted through telemedicine only, physical consultations took place in the form of a clinical examination at the practice (9.7\%), a PCR on nasopharyngeal swab $(12.4 \%)$ and an emergency services consultation (1.8\%). Figure 2 shows these results. At the end of the telemedicine consultation, medication was prescribed in $12.5 \%$ of cases. Treatment with a bronchodilator was prescribed in $3 \%$ of cases and an antibiotic in $0.5 \%$ of cases. Sick leave was required for $17.5 \%$ of telephone calls.

The telephone calls lasted on average 7.85 minutes (standard deviation 3.07 minutes). The number of calls halved starting from week 19 . Figure 3 shows the distribution of calls by day of the week. Telephone calls were more frequent on Mondays (24\%) and Fridays (18.5\%).

\section{Description of symptoms}

During the 44 telephone calls for initial assessment, patients used to describe a dry cough (54.5\%), fatigue (50\%), sore throat (47.7\%), headache (45.5\%), fever (45.4\%). Figure 4 shows the different symptoms mentioned by patients during the initial assessment.

Figure 5 shows the evolution of patient symptoms over time. $60 \%$ of telephone calls for initial assessment took place within the first 4 days of onset of symptoms. $65 \%$ of calls indicated an improvement on D12. A worsening was described in 8\% of calls between D5 and D8 and in $15 \%$ of calls from day 9 of symptoms. At 3 weeks (> D-22) 6 telephone calls still described stable symptoms, corresponding to $13.6 \%$ of the number of initial calls.

\section{Discussion}

\section{Study methodology}

To the best of our knowledge, this is the first study carried out in a primary care setting within a private practice and not in a hospital outsetting. Our study began on 6 April 2020 (week 15), i.e. one week after the epidemic peak in the Canton of Fribourg (8). The number of calls gradually decreased from week 18 and we concluded the study at the end of May due to the fall in the number of telephone calls. Switzerland was among the countries with the highest number of cases in Spring 2020 in Europe (9). Switzerland has not had a strict lockdown policy and the majority of the restrictive measures were lifted on 11.05.2020, with the exclusion of the social distancing directives, which are still in force (10).

\section{Telemedicine assistance}

The assistance for most patients (73\%) only required one telephone call. During this period, the recommendations by the Swiss authorities did not include the performance of systematic PCR swabs, which explains the low number of consultations based on this reason. Today, the results would be different due to the current broad testing policy. In addition, over the entire follow-up, only $23.9 \%$ of patients required a physical consultation, most often for carrying out a COVID-19 PCR swab $(12.4 \%$ of 
patients). Patient described a worsening of symptoms in $15 \%$ of cases from the $9^{\text {th }}$ day of symptoms, and stability of symptoms in $30 \%$ of cases. Post-acute covid-19 (or "long covid") has been described in the literature and appears to occur in approximately $10 \%$ of cases (11). We also found $13.6 \%$ of telephone calls which were still symptomatic calls after 3 weeks.

The assistance consisted mainly in the advice of isolation, monitoring and quarantine, while only $12.5 \%$ of the calls required a prescription. This low number is explained by the fact that many patients already had paracetamol at home and currently there is no specific treatment for COVID-19 in the outpatient setting. The prescription of antibiotics is low in our study, which is probably due to the fact that they are ineffective in the treatment of COVID-19, and patients know and accept this.

Interestingly, only $17.5 \%$ of calls required a sick leave certificate. This shows how the calls were about the monitoring of a sick population and not the management of administrative problems.

It is also interesting to observe that the calls were mainly from a young population, with an average age of 44 years old. We had expected different results, in particular an older population and an increased need for physical consultations. In our opinion, these results confirm the effectiveness of social distancing measures and of the recommendations for the shielding of people defined as to be at risk, who therefore, probably have had less contact with the virus compared to the younger working population.

We could not use videoconferencing for the COVID-19-related assistance of patients, because it would have been too complicated to set up the device and this would have been less accessible to some patients. However, as several authors have mentioned, this could be an option for the assessment of patients, mainly young and having access to such technology, which is appreciated by patients $(3,12)$.

\section{Symptoms}

Despite having carried out a temporal collection of symptoms, we cannot say for sure that they suffered from a COVID-19 infection, given the low number of PCR tests carried out. A review has shown that it is not possible to distinguish a Covid-19 infection from another virus infection only on the basis of the symptoms (7).

\section{Organisational aspects}

The number of telephone calls was higher on Mondays (24.2\% of cases), which required more staff on that day. There were fewer calls on Saturdays and Sundays, which is explained by the fact that the medical centre is closed. However, it is possible that on weekends there were more telephone calls directed to the hotlines. Despite the fact that the medical centre had no previous experience of telemedicine consultations, we were not overwhelmed by the handling of these calls, which lasted less than 10 minutes. In our opinion, these results were possible because the physicians who carried out the telephone follow-up were the patients' family doctors, and/or had access to the patient's medical file. 
We believe that this in-depth knowledge of the patient allowed higher quality assistance and restricted the need for consultations. We also observed that the glossary facilitated the identification of the different situations and made our assistance more consistent. The glossary also allowed the researchers to follow the evolution of the recommendations for assistance, in particular in relation to carrying out nasopharyngeal PCR swabs.

\section{Conclusion}

This study shows that a rigorous telephone follow-up strategy implemented by primary care physicians requires few physical consultations in patients with flu-like symptoms in the context of the COVID-19 epidemic. Telemedicine assistance generates very few drug prescriptions, in particular, negligible antibiotics. For the establishment of telemedicine consultations in general-practitioner practices, we recommend increasing work capacities on Mondays and that all physicians carrying out telephone consultations use a regularly updated summary document, identifying the situations and the recommendations for the assistance.

\section{Declarations}

\section{Acknowledgements}

We would like to thank the researchers: Christelle Clerc, Myriam Perret, Josephine Tornay and Noëmí Zurron.

We would also like to thank all the staff of the La Tour medical practice who supported the study.

\section{Authors' contributions}

The study was conceived of and designed by J. Suillot, J. Sagez and S. Zuercher. Data was analysed by J. Suillot and J. Sagez. The report was drafted by J. Sagez and J. Suillot and was critically revised by all the authors. All authors read and approved the final report.

\section{Funding}

The researchers did not receive any additional remuneration for their work. Organisational costs were entirely financed by the La Tour medical practice.

\section{Availability of data and materials}

The relevant datasets are available from the corresponding author Julien Sagez (js@cm-latour.ch) upon reasonable request.

\section{Ethics approval and consent to participate}


Patient consent was obtained with each call. The data was collected anonymously. The study obtained the prior authorisation of the cantonal commission (VD) for ethics in the field of research on human beings (CER-VD) (Project 2020-00595).

\section{Consent for publication}

Not applicable

\section{Competing interests}

The authors declare that they have no competing interests.

\section{Author details}

${ }^{1}$ La Tour medical practice, 1635 La Tour-de-Trême, Switzerland

${ }^{2}$ Department of Internal Medicine, Fribourg Hospital, Switzerland.

${ }^{3}$ University of Fribourg, Switzerland

\section{References}

1. WHO. 2020 [Available from: https://www.who.int/docs/default-source/coronaviruse/situationreports/20200311-sitrep-51-covid-19.pdf?sfvrsn=1ba62e57_10.

2. Lim WH, Wong WM. COVID-19: Notes From the Front Line, Singapore's Primary Health Care Perspective. Ann Fam Med. 2020;18(3):259-61.

3. Greenhalgh T, Koh GCH, Car J. Covid-19: a remote assessment in primary care. BMJ. 2020;368:m1182.

4. Doshi A, Platt Y, Dressen JR, Mathews BK, Siy JC. Keep Calm and Log On: Telemedicine for COVID-19 Pandemic Response. J Hosp Med. 2020;15(5):302-4.

5. Crane SJ, Ganesh R, Post JA, Jacobson NA. Telemedicine Consultations and Follow-up of Patients With COVID-19. Mayo Clin Proc. 2020;95(9S):S33-S4.

6. Huston P, Campbell J, Russell G, Goodyear-Smith F, Phillips RL, Jr., van Weel C, et al. COVID-19 and primary care in six countries. BJGP Open. 2020;4(4).

7. Struyf T, Deeks JJ, Dinnes J, Takwoingi Y, Davenport C, Leeflang MM, et al. Signs and symptoms to determine if a patient presenting in primary care or hospital outpatient settings has COVID-19 disease. Cochrane Database Syst Rev. 2020;7:CD013665.

8. FR.ch. 2020 [Available from: https://www.fr.ch/de/gesundheit/covid-19/coronavirus-statistik-ueberdie-entwicklung-im-kanton.

9. Salathe M, Althaus CL, Neher R, Stringhini S, Hodcroft E, Fellay J, et al. COVID-19 epidemic in Switzerland: on the importance of testing, contact tracing and isolation. Swiss Med Wkly. 
2020;150:w20225.

10. BAG. 2020 [Available from: https://www.bag.admin.ch/bag/de/home/krankheiten/ausbruecheepidemien-pandemien/aktuelle-ausbrueche-epidemien/novel-cov/situation-schweiz-undinternational.html.

11. Greenhalgh T, Knight M, A'Court C, Buxton M, Husain L. Management of post-acute covid-19 in primary care. BMJ. 2020;370:m3026.

12. Smith WR, Atala AJ, Terlecki RP, Kelly EE, Matthews CA. Implementation Guide for Rapid Integration of an Outpatient Telemedicine Program During the COVID-19 Pandemic. J Am Coll Surg. 2020;231(2):216-22 e2.

\section{Tables}

\section{Inclusion criteria}

1. Calls from patients showing symptoms suggestive of COVID-19 (upper and lower airway infection, fever, dysgeusia, anosmia, asthenia)

2. Patient telephone calls for advice regarding COVID-19

3. Patient over 18 years old

1. Patient previously followed at the practice in adult general medicine

j. Participation consent

Exclusion criteria

1. Patient less than 18 years old

2. Patient not registered with the practice for adult general medicine

3. Impediment to communication or telephone consultation not possible (deafness, foreign language, etc.)

Table 1: Inclusion and exclusion criteria 
- First consultation or follow-up consultation

- Consultation on patient request or scheduled consultation

- Age and gender of patient

- Duration of the telephone call

Description of symptoms

1. Dating of symptoms

2. Evolution of symptoms: improvement, stability, worsening or recovery

3. Symptoms on the day of the telephone call (fever, cough, sputum production, haemoptysis, myalgia or arthralgia, fatigue, nasal symptoms, sore throat, dyspnoea, chest pain, chest burn, headache, anosmia or ageusia, gastrointestinal symptoms.)

Description of the assistance provided

1. Modality of consultation (telemedicine, physical consultation at the practice, emergency services consultation)

2. Isolation recommendation

3. Prescription

1. Completion of a certificate of incapacity for work

ว. Scheduled telephone follow-up or on the request of the patient

j. Recommendation of a PCR test

Table 2: Data collected during the telephone interview

\section{Figures}




\section{A}

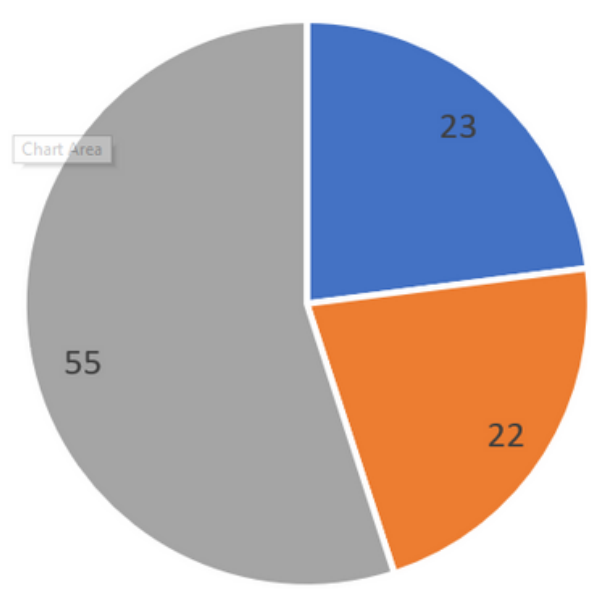

- Information and advise " Fisrt call for symptoms - Follow-up

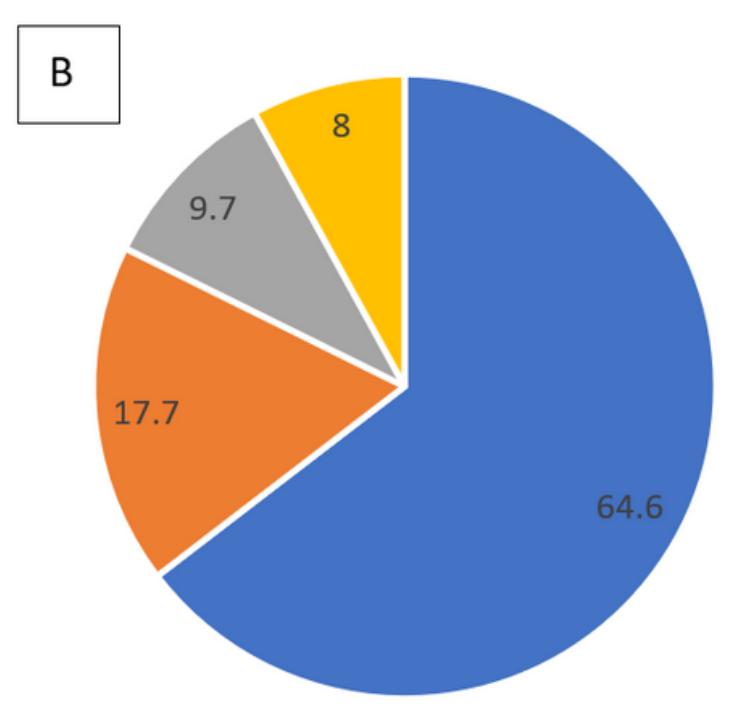

- $1-2=3->3$

\section{Figure 1}

A. Reason for calling $(\mathrm{N}=200)$ and $B$. Number of calls by patient $(\mathrm{N}=113)$.

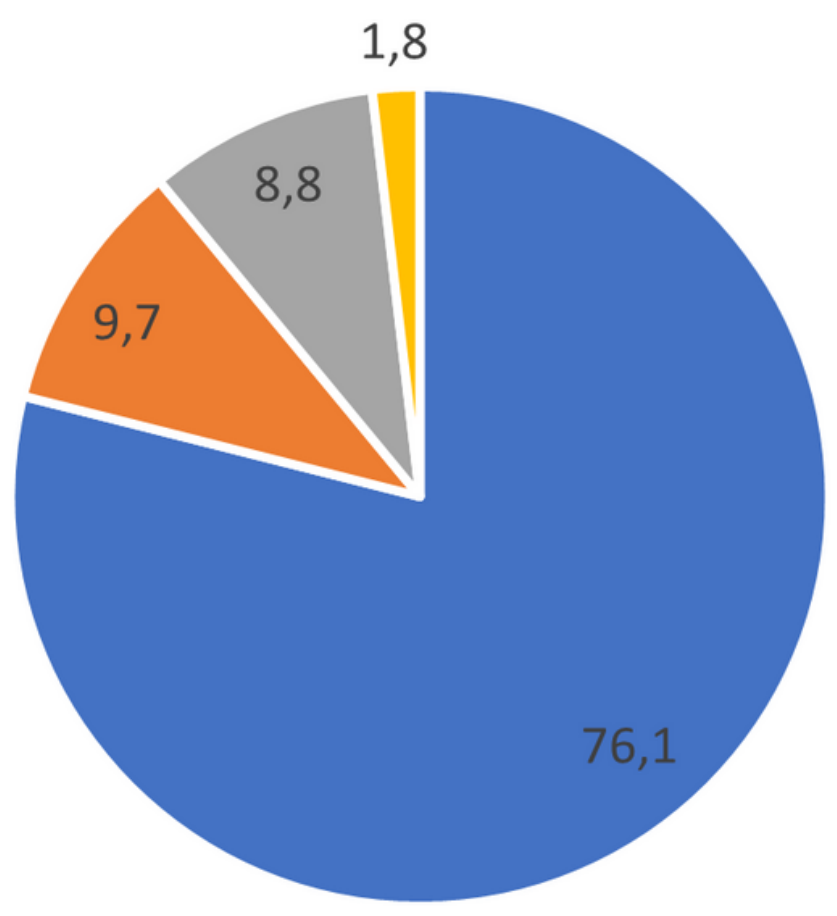

- Telemedecine

- Consultation for PCR
- Consultation for physical evaluation

- Emergency 
Figure 2

Modality of consultation carried out for the 113 patients.

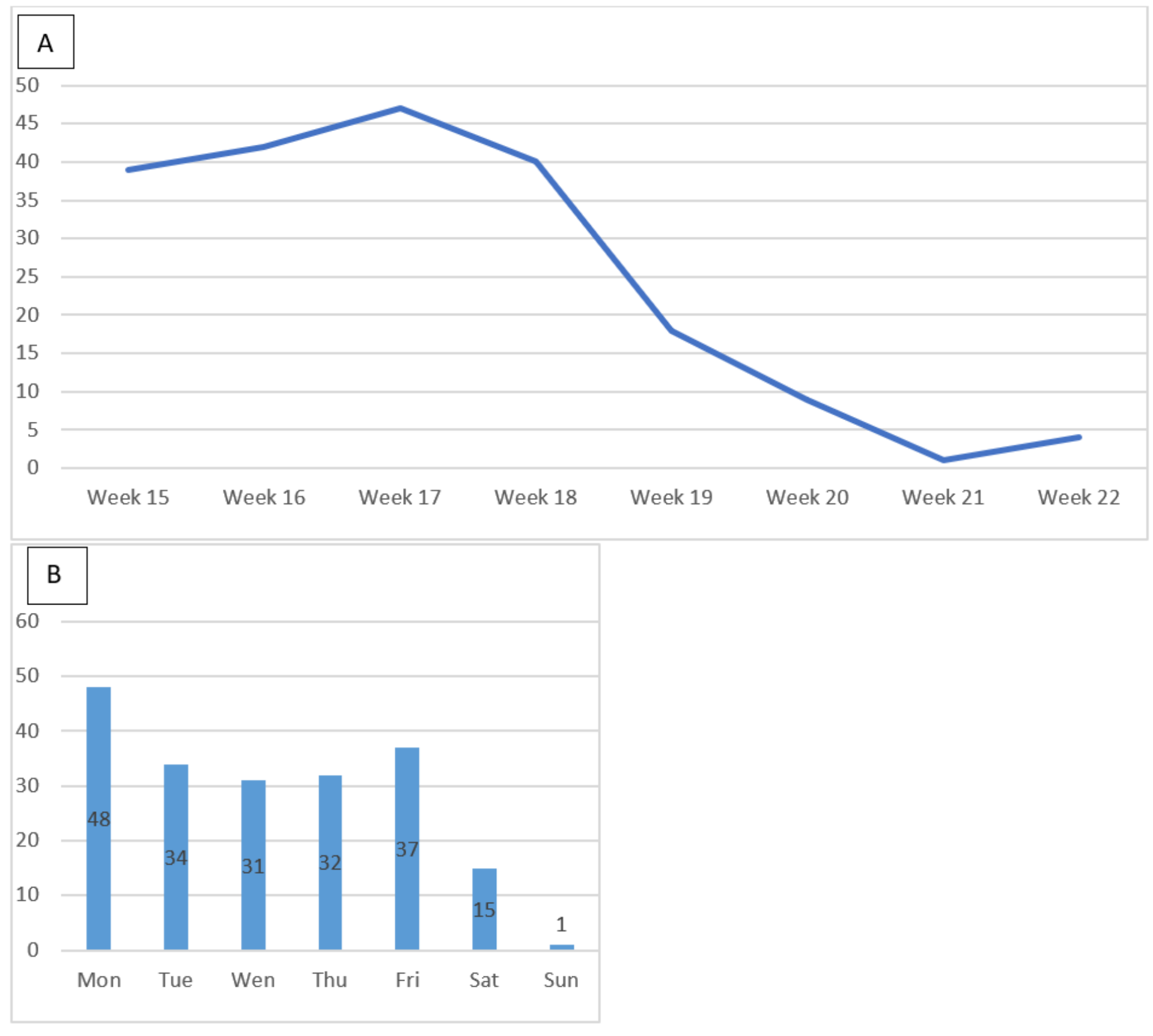

\section{Figure 3}

A. Evolution of the number of telephone calls per week during the study. B. Distribution of calls by day of the week. $\mathrm{N}=200$. 




\section{Figure 4}

Symptoms described by the patient on the first call. Values are in percentage. $\mathrm{N}=44$.

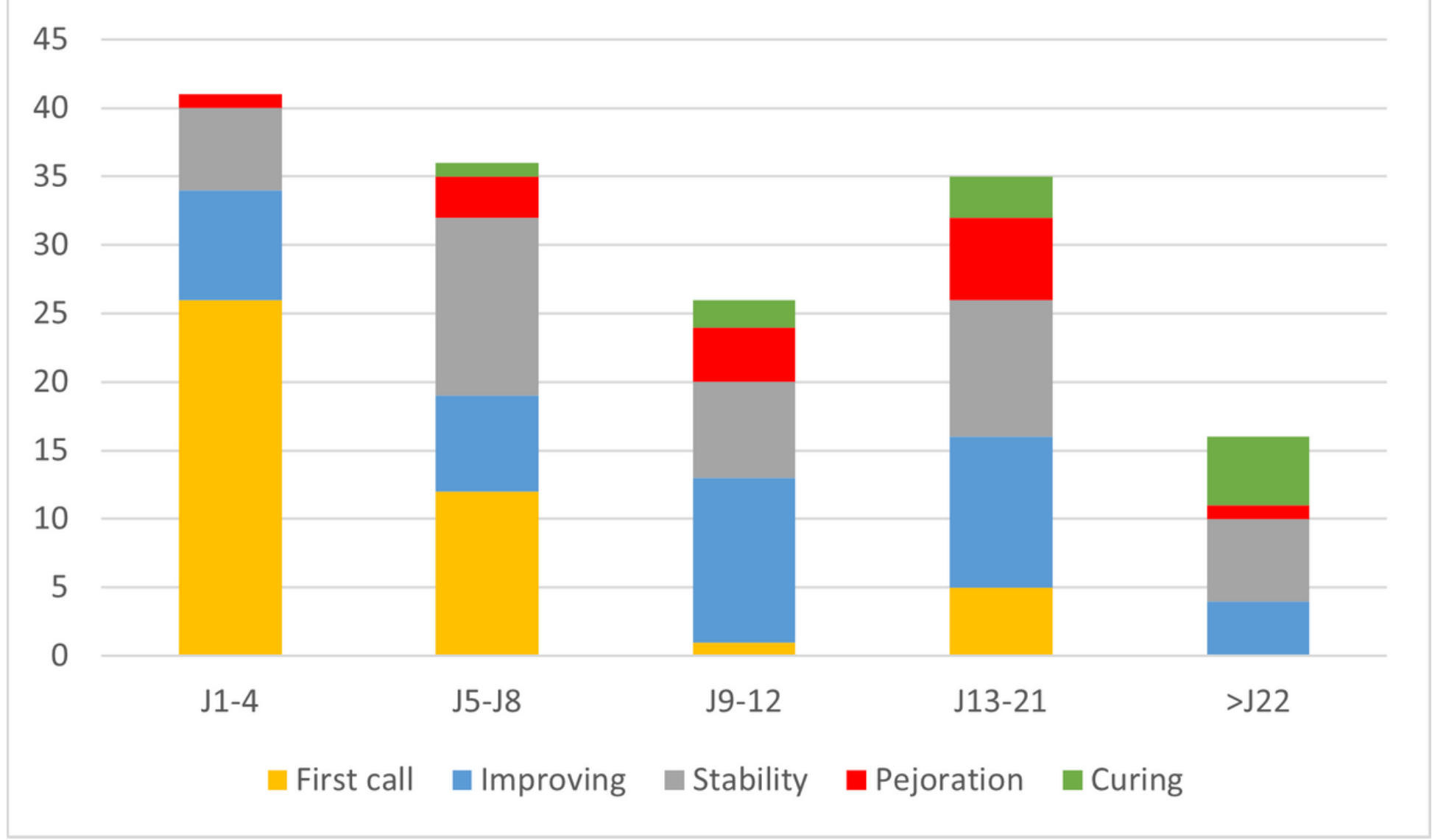

\section{Figure 5}

Telephone calls of symptomatic patients. $\mathrm{N}=154$. 


\section{Supplementary Files}

This is a list of supplementary files associated with this preprint. Click to download.

- RsultatCOVIDCM202007301.xIsx 